\title{
ORIGINAL
}

\section{DERIVACIONES EN LOS CENTROS DE SALUD DE ANDALUCÍA SEGÚN EL SEXO DE PROFESIONALES Y PACIENTES. UN ANÁLISIS DE GÉNERO (*)}

\author{
Ana Delgado (1), Lorena Saletti-Cuesta (1), Carmen Sánchez-Cantalejo (1), Begoña \\ López-Hernández (2), Pilar Guijosa-Campos (2), Margarita Acosta-Ferrer (3), Juana \\ Montoya-Vergel (4) y Natalia Gil-Garrido (5).
}

\author{
(1) Escuela Andaluza de Salud Pública. \\ (2) Distrito Sanitario Granada-Metropolitano. \\ (3) Distrito Sanitario Almería. \\ (4) Distrito Sanitario Poniente de Almería. \\ (5) Centro de salud de Iznájar. Distrito Sanitario Córdoba Sur.
}

(*) Fondo de Investigación Sanitaria (Expediente PI10/00124).

Sin conflictos de intereses.

\section{RESUMEN}

Fundamentos: En atención primaria se han identificado diferencias de práctica según sexo del profesional y, simultáneamente, existen desigualdades de género en la asignación de recursos sanitarios, aspectos ambos que suscitan un interés creciente en la gestión y provisión de servicios de salud. El objetivo del estudio es conocer si existen diferencias de práctica en las derivaciones sanitarias realizadas por médicas y médicos de familia (MF) de centros de salud de Andalucía, si existen desigualdades en las derivaciones recibidas por hombres y mujeres, y si existe interacción sexo de profesional sexo de paciente.

Métodos: Estudio transversal y multicéntrico. Población: MF de 4 distritos sanitarios (DDSS). Muestra: $382 \mathrm{MF}$. Variables: tasa de derivaciones por visita (TDxV), tasa de derivaciones por cupo (TDxC), sexo de paciente; sexo, edad, y formación postgraduada en medicina familiar de MF, tamaño del cupo por sexo, media de visitas /paciente por sexo, edad media del cupo por sexo, y proporción de hombres en el cupo; centro de salud urbano/rural, tamaño del equipo, población adscrita y acreditación docente. Fuentes: bases de datos de los DDSS. Análisis estadístico: descriptivo. Bivariante y multivariante mediante análisis multinivel de la TDxV con modelo mixto de Poisson.

Resultados: En 2010 los/as 382 MF realizaron 129.161 derivaciones a especialistas. La TDxC fue 23,47 y la TDxV es 4,92. Las TDxC de las médicas fue 27,23 en mujeres y 19,78 en hombres y las de los médicos 27,37 en mujeres y 19,51 en hombres. La TDxV de las médicas fueron 4,92 en mujeres y 5,48 en hombres y para los médicos 4,54 y 4,93 respectivamente.

Conclusiones: No existen diferencias en las derivaciones según sexo de MF, sin embargo, existen indicios de que las mujeres son menos derivadas. No existe interacción sexo profesional-sexo paciente.

Palabras clave: Derivación y consulta. Práctica profesional. Atención primaria de salud. Género y salud.

Correspondencia

Ana Delgado Sánchez

C/Alquería, 66

18010 Granada

ana.delgado.easp@juntadeandalucia.es

\section{ABSTRACT \\ Referrals and Sex of Physicians and Patients. A Gender Analysis, Andalussia, Spain}

Background: It has been identified differences of medical care practice in primary care related to physician's sex. Simultaneously, there are gender inequalities in the assignment of health resources. Both aspects give rise to an increasing growing interest in the management and provision of health services. Objectives: To examine the differences in the referral practice made by female and male primary care physicians working in health centers in Andalusia, to consider whether there are disparities in referrals received by men and women, and to examine the interaction between patient's sex and physician's sex.

Methods: Observational, cross-sectional, and multicenter study. Population: 4 health districts in Andalucía and their physicians. Sample: 382 physicians. Measurements: referral rate per visit (RV), referral rate per patient quota (RQ), patient's sex, physician: sex, age, postgraduate family medicine specialty, size of the patient quota by sex, mean number of patients/day by sex, mean age of the patient quota by sex, and proportion of men in the quota. Health center: urban / rural, size of the team, enrolled population, and postgraduate family medicine specialty's accreditation. Sources: databases of health districts. Period of study: 2010. Analysis: Bivariate and multivariate multilevel analysis of the referral rate per visit with mixed Poisson model.

Results: In 2010382 physicians made 129,161 referrals to specialized care. The RQ was 23.47 and the RV was 4.92 . The RQ in women and men was 27.23 and 19.78 for women physicians, being 27.37 and 19.51 for male physicians. The RV in women and men was 4.92 and 5.48 for women physicians, being 4.54 and 4.93 for male physicians.

Conclusion: There are no differences in referral according to physician's sex. However, there are signs that might indicate the existence of gender inequality, and women patient received less referrals. There are no physician-patient's sex interaction.

Keyword: Referral and Consultation. Professional practice. Primary health care.Gender and Health 


\section{INTRODUCCIÓN}

La derivación es un proceso de relación formal entre profesionales de distintos niveles asistenciales para la atención de un paciente $^{1,2}$. En los países como España, donde médicos y médicas de familia tienen la función de filtro (gatekeeper), los pacientes no pueden acceder directamente al nivel hospitalario, son estos profesionales quienes identifican la necesidad de la derivación y la autorizan, decidiendo así sobre la asignación de recursos sanitarios que satisfagan las demandas de pacientes ${ }^{1,3}$. La información sobre las derivaciones entre niveles permite a gestores y profesionales sanitarios identificar las variables que influyen en el proceso, anticipar la demanda de servicios y mejorar la equidad en la distribución de los recursos sanitarios ${ }^{2}$.

En la tasa de derivaciones existe variabilidad que viene explicada por características del sistema sanitario, de profesionales y de pacientes, aunque los resultados de los estudios son dispares. En España y en otros países la tasa de derivaciones se asocia al hábitat urbano/rural por su relación con la accesibilidad geográfica al nivel especializado ${ }^{4,5}$ y la formación o a la edad del profesional $1,2,5-7$.

Las diferencias de práctica clínica según género, relacionadas sobre todo con el estilo de comunicación durante la consulta, son bien conocidas ${ }^{8,9}$ pero en el caso de las derivaciones los resultados son poco consistentes. Según unos estudios las médicas derivan más que los médicos ${ }^{2,4}$ y según otros no existen diferencias ${ }^{10}$.

Desde el punto de vista de pacientes se ha observado que el factor más determinante de la derivación es la morbilidad,11,12. También su sexo afecta a la tasa de derivaciones, siendo, según unos estudios más derivadas las mujeres ${ }^{2,13}$, según otros los hombres ${ }^{10,14}$ $\mathrm{y}$, por último, hay hallazgos que no muestran diferencias entre ambos ${ }^{5,7,11}$. Se sabe que las desigualdades de género en la asistencia sanitaria, o sesgos de género, afectan a numerosas situaciones clínicas ${ }^{15,16}$, siendo la más conocida la enfermedad coronaria ${ }^{17}$. Los sesgos de género más estudiados hasta la fecha señalan situaciones de desventaja para las mujeres, pero existen resultados sobre sesgos que afectan a los hombres, por ejemplo en el caso de la migraña ${ }^{18}$. Todo ello produce inequidad en la prestación de cuidados de salud.

Los objetivos de este estudio son: a) conocer si existen diferencias de práctica en las derivaciones hospitalarias realizadas por médicas y médicos de familia que trabajan en centros de salud de Andalucía, b) conocer si existen desigualdades de género en las derivaciones realizadas a hombres y mujeres que consultan en los mismos centros de salud y c) conocer si existe interacción sexo de profesional-sexo de paciente.

\section{MATERIAL Y MÉTODOS}

Diseño. Realizamos un estudio transversal y multicéntrico. La población de referencia fueron 708 profesionales de medicina de familia (MF) de 58 centros de salud (CS) de 4 Distritos Sanitarios (DDSS) de Andalucía. Los CS se clasificaron según la isocrona al hospital de referencia $(\leq 20$ minutos o $>20$ minutos). El criterio de selección fue que se llevara trabajando como mínimo un año con el mismo cupo de pacientes. Cumplían el criterio 396 MF, se excluyó a 14 MF por problemas en el registro, quedando una muestra definitiva de $382 \mathrm{MF}$.

Variables. La variable dependiente fue el número de primeras derivaciones, totales $\mathrm{y}$ por especialidad, excluyendo las de causa gineco-obstétrica y urológica, esta última por ser la patología urológica mayoritariamente de origen prostático. No se incluyeron las derivaciones para pruebas de imagen ni las revisiones. Las covariables fueron: sexo del/la paciente. De profesionales: sexo, edad, formación posgraduada en 
medicina familiar y comunitaria (MIRMF), número de pacientes en el cupo en junio de 2010 desagregado por sexo, media de visitas /día en consulta a demanda durante el año 2010 para cada sexo, edad media de los hombres y de las mujeres en el cupo, y proporción de hombres en el cupo. De CS: hábitat urbano/rural, tamaño del equipo de atención primaria (enfermería, medicina de familia y pediatría), tamaño de la población adscrita y si tenía o no acreditación docente para formación MIRMF.

La información sobre derivaciones hospitalarias procedió de la base de datos MTICITAS de los DDSS, la cual no proporciona el motivo o diagnóstico que ocasiona la derivación. Así mismo los DDSS proporcionaron la población (MTI-BDU) y la información sobre profesionales Gestión y Archivo de datos de Usuario (GADU).

El período estudiado fue el año 2010 . La recogida de datos se hizo entre enero de 2011 y julio de 2012 .

Construimos dos variables dependientes para el análisis de las derivaciones al hospital: tasa de derivaciones ajustada por cupo (TDxC) y tasa de derivaciones ajustada por número de visitas o frecuentación (TDxV), y seleccionamos la TDxV como variable final, ya que la derivación se producía durante una visita y la probabilidad de que ocurriera guardaba relación con la frecuentación de cada persona, siendo la media de visitas/año 6,53 en mujeres y 4,25 en hombres.

Análisis estadístico. Descripción de todas las variables del estudio mediante medidas de resumen. Dada la estructura de la muestra en tres niveles, paciente, profesional y $\mathrm{CS}$, los datos se analizaron con un modelo de efectos aleatorios cuyas componentes aleatorias eran CS y MF y cuya parte de efectos fijos correspondió a las covariables estudiadas, aplicándose el modelo al análisis bivariante y multivariante. Al incluir covariables del CS que estaban fuertemente asociadas con su factor de efectos aleatorios, la varianza estimada del CS no fue diferente de cero significativamente, por lo que el nivel CS desaparecía quedando un modelo con solo dos niveles.

Para el análisis multinivel de las TDxV se ajustó un modelo mixto de Poisson para la variable dependiente "número de derivaciones" y exposición "número de citas, con el objetivo de modelizar derivaciones por cita. El método utilizado fue el de máxima verosimilitud restringida y los resultados obtenidos para la medida natural del modelo fueron la razón de tasas de incidencia (IRR) y sus intervalos de confianza al 95\%. El modelo ajustado se hizo con todas las variables estudiadas con el fin de controlar por su conjunto. Se obtuvo el IRR para medir el efecto del MF como factor de efectos aleatorios, medida que comparaba las tasas de incidencias entre el MF que más derivaba y el que menos. La interacción sexo profesional-sexo paciente se ajustó en todos los modelos y se hizo un análisis a posteriori de las cuatro díadas de interés para obtener todas las comparaciones posibles.

Los programas empleados fueron Stata 12.1 y xtmepoisson.

\section{RESULTADOS}

Durante el año 2010 se realizaron 129.161 derivaciones a hospitales por $382 \mathrm{MF}$ que trabajaban en 58 CS de los 4 DDSS estudiados.

Las tablas 1 y 2 contienen las características de la muestra, destacando que 156 (41\%) eran médicas, 24 (41\%) CS estaban a más de 20 minutos de su hospital de referencia, las mujeres hacían casi 2 visitas/año más que los hombres, la tasa de derivaciones ajustada por cupo fue 23,47, y ajustada por visita 4,92. En las mujeres fue mayor que en hombres la TDxC $(27,31 v s 19,62)$ y menor la TDxV $(4,69$ vs 5,15$)$. 
Tabla 1

Descriptiva de las características cualitativas de las variables estudiadas

\begin{tabular}{|c|c|c|c|}
\hline & Categoría & $\mathrm{N}$ & Porcentaje \\
\hline \multicolumn{4}{|c|}{ Centro de Salud $(\mathrm{n}=58)$} \\
\hline \multirow{2}{*}{ Acreditación docente para formación MIRMF } & No & 33 & 56.9 \\
\hline & $\mathrm{Si}$ & 25 & 43,1 \\
\hline \multirow{2}{*}{ Ámbito } & Rural & 24 & 41,4 \\
\hline & Urbano & 34 & 58,6 \\
\hline \multicolumn{4}{|c|}{ Profesional $(\mathrm{n}=382)$} \\
\hline \multirow{2}{*}{ Sexo } & Mujer & 156 & 40,8 \\
\hline & Hombre & 226 & 59,2 \\
\hline \multirow{2}{*}{ Formación MIRMF } & Sí & 134 & 35,1 \\
\hline & No & 248 & 64,9 \\
\hline \multirow{2}{*}{ Trabaja en Centro de Salud acreditado para formación MIR } & No & 190 & 49,7 \\
\hline & Sí & 192 & 50,3 \\
\hline \multirow{4}{*}{ Distrito Sanitario } & 1 & 149 & 39 \\
\hline & 2 & 53 & 13,9 \\
\hline & 3 & 61 & 16 \\
\hline & 4 & 119 & 31,2 \\
\hline \multirow{2}{*}{ Trabaja en ámbito } & Rural & 126 & 33 \\
\hline & Urbano & 256 & 67 \\
\hline
\end{tabular}

MIRMF: Formación postgraduada en Medicina Familiar y Comunitaria

Tabla 2

Descriptiva de las características cuantitativas de las variables estudiadas

\begin{tabular}{|c|c|c|}
\hline & Media & Desviación típica \\
\hline \multicolumn{3}{|c|}{ Centro de Salud $(\mathrm{n}=58)$} \\
\hline Población atendida & $15.820,28$ & $7.965,68$ \\
\hline Tamaño del equipo & 20,19 & 9,002 \\
\hline \multicolumn{3}{|c|}{ Profesional (n=382) } \\
\hline Edad & 53,87 & 5,37 \\
\hline Edad media cupo pacientes Hombres & 43,46 & 3,54 \\
\hline Edad media cupo pacientes Mujeres & 45,78 & 4,17 \\
\hline Tamaño del cupo de hombres & 715,23 & 165,01 \\
\hline Tamaño del cupo de mujeres & 736,13 & 152,39 \\
\hline Número de visitas de hombres & $2.957,01$ & 819,70 \\
\hline Número de visitas de mujeres & $4.742,09$ & $1.436,55$ \\
\hline Media de visitas por hombre & 4,25 & 1,25 \\
\hline Media de visitas por mujer & 6,53 & 1,86 \\
\hline Tasa de derivaciones $\mathrm{x} 100$ personas & 23,47 & 8,18 \\
\hline Tasa de derivaciones por 100 hombres & 19,62 & 5,89 \\
\hline Tasa de derivaciones por 100 mujeres & 27,31 & 8,34 \\
\hline Tasa de derivaciones por 100 visitas & 4,92 & 4,05 \\
\hline Tasa de derivaciones por 100 visitas en hombres & 5,15 & 4,30 \\
\hline Tasa de derivaciones por 100 visitas en mujeres & 4,69 & 3,78 \\
\hline
\end{tabular}


Tabla 3

Comparación de las variables de estudio entre médicas y médicos

\begin{tabular}{|c|c|c|c|}
\hline Variables cuantitativas & Médicas & Médicos & $\mathbf{p}$ \\
\hline Edad & 52,45 & 54,85 & $<0,001$ \\
\hline Edad media cupo hombres & 43,49 & 43,44 & 0,890 \\
\hline Edad media cupo mujeres & 45,23 & 46,16 & 0,033 \\
\hline Tamaño del cupo & $1.471,33$ & $1.437,57$ & 0,227 \\
\hline Tamaño del cupo de hombres & 694.58 & 729.48 & 0,033 \\
\hline Tamaño del cupo de mujeres & 776.75 & 708.09 & $<0,001$ \\
\hline Número total de visitas & 7.748 .94 & 7.664 .92 & 0,707 \\
\hline Número de visitas de hombres & 2.846 .92 & 3.032 .52 & 0,030 \\
\hline Número de visitas de mujeres & 4.902 .03 & 4.632 .39 & 0,072 \\
\hline Tasa de derivaciones $\times 100$ personas & 23,51 & 23,44 & 0,917 \\
\hline Tasa de derivaciones por 100 hombres & 19,78 & 19,51 & 0,922 \\
\hline Tasa de derivaciones por 100 mujeres & 27,23 & 27,37 & 0,946 \\
\hline Tasa de derivaciones por 100 visitas & 5,20 & 4,73 & 0,666 \\
\hline Tasa de derivaciones por 100 visitas en hombres & 5,48 & 4,93 & 0,514 \\
\hline Tasa de derivaciones por 100 visitas en mujeres & 4,92 & 4,54 & 0,530 \\
\hline Variables cualitativas & Médicas & Médicos & $\mathbf{p}$ \\
\hline Trabajan en Centro de salud urbano & $66,7 \%$ & $67,3 \%$ & 0,900 \\
\hline Trabajan en Centro de salud acreditado para formación MIRMF & $51,3 \%$ & $49,6 \%$ & 0,74 \\
\hline Tienen formación MIRMF & $34 \%$ & $35,8 \%$ & 0,71 \\
\hline
\end{tabular}

MIRMF: Formación postgraduada en Medicina Familiar y Comunitaria

Tabla 4

Modelos Crudo y Ajustado para la tasa de derivaciones por visitas (TDxV)

\begin{tabular}{|c|c|c|c|c|c|c|c|c|c|}
\hline \multirow{2}{*}{ Variables } & \multirow{2}{*}{ Categorias } & \multirow{2}{*}{ Media } & \multirow{2}{*}{$\begin{array}{c}\text { Desviación } \\
\text { típica }\end{array}$} & \multicolumn{3}{|c|}{ Modelo crudo } & \multicolumn{3}{|c|}{ Modelo ajustado } \\
\hline & & & & IRR & $\mathrm{IC}$ & $\mathrm{p}$ & IRR & $\mathrm{IC}$ & $\mathrm{p}$ \\
\hline Población asignada & & & & 1 & $1-1$ & 0,791 & 1 & 11 & 0,317 \\
\hline Tamaño del equipo & & & & 1 & $1-1$ & 0,932 & 1 & $0,99-1,01$ & 0,758 \\
\hline Edad media MF & & & & 1 & $0,99-1,01$ & 0,821 & 1 & $0,99-1,01$ & 0,483 \\
\hline Edad media paciente & & & & 0,98 & $0,98-0,99$ & 0,000 & 1,01 & $1,01-1,02$ & 0,000 \\
\hline \%de hombres en el cupo & & & & 1,01 & $1-1,01$ & 0,155 & 0,99 & $0,98-1$ & 0,075 \\
\hline \multirow{4}{*}{ Distrito sanitario } & 1 & 0,037 & 0,009 & 1 & & & 1 & & \\
\hline & 2 & 0,048 & 0,035 & 1,14 & $1,01-1,28$ & 0,040 & 1,23 & $1,03-1,48$ & 0,025 \\
\hline & 3 & 0,051 & 0,015 & 1,37 & $1,22-1,54$ & 0,000 & 1,66 & $1,43-1,94$ & 0,000 \\
\hline & 4 & 0,065 & 0,064 & 1,55 & $1,41-1,70$ & 0,000 & 1,69 & $1,52-1,89$ & 0,000 \\
\hline \multirow{2}{*}{$\begin{array}{l}\text { Acreditacón } \\
\text { IRMF }\end{array}$} & No & 0,051 & 0,053 & 1,00 & & & 1 & & \\
\hline & Sí & 0,048 & 0,021 & 1,01 & $0,92-1,1$ & 0,850 & 0,95 & $0,87-1,04$ & 0,249 \\
\hline \multirow{2}{*}{ Hábitat } & Rural & 0,055 & 0,046 & 1 & & & 1 & & \\
\hline & Urbano & 0,046 & 0,020 & 0,93 & $0,85-1,02$ & 0,119 & 1,08 & $0,95-1,21$ & 0,243 \\
\hline \multirow{2}{*}{ Sexo paciente } & Hombre & 0,052 & 0,043 & & & & & & \\
\hline & Mujer & 0,047 & 0,038 & 0,91 & $0,90-0,92$ & 0,000 & & & \\
\hline \multirow{2}{*}{ Sexo MF } & Hombre & 0,047 & 0,022 & 1 & & & & & \\
\hline & Mujer & 0,052 & 0,057 & 1,02 & $0,93-1,11$ & 0,666 & & & \\
\hline \multirow{4}{*}{$\begin{array}{l}\text { Díadas } \\
\text { MF-paciente }\end{array}$} & Médico- Hombre & 0,049 & 0,022 & 1 & & & 1 & & \\
\hline & Médico- Mujer & 0,045 & 0,023 & 0,91 & $0,90-0,93$ & 0,000 & 0,88 & $0,86-0,90$ & 0,000 \\
\hline & Médica- Hombre & 0,055 & 0,062 & 1,03 & $0,94-1,13$ & 0,515 & 0,99 & $0,91-1,08$ & 0,871 \\
\hline & Médica- Mujer & 0,049 & 0,052 & 0,95 & $0,85-1,02$ & 0,108 & 0,87 & $0,80-0,95$ & 0,002 \\
\hline \multirow{2}{*}{$\begin{array}{l}\text { Formación postgraduada } \\
\text { Medicina de Familia }\end{array}$} & No & 0,051 & 0,047 & 1,00 & & & 1 & & \\
\hline & Sí & 0,046 & 0,023 & 0,95 & $0,87-1,04$ & 0,258 & 1,04 & $0,95-1,14$ & 0,444 \\
\hline
\end{tabular}


En la tabla 3 destaca que las médicas eran más jóvenes $(52,45$ vs $54,85 \mathrm{p}<0,001)$ y tenían más mujeres en el cupo que los médicos $(52,8 \%$ vs $49,3 \% \mathrm{p}<0,001)$. Tanto médicas como médicos tenían TDxC más elevadas en mujeres que en hombres (médicas 27,23 vs 19,78 y médicos 27,37 vs 19,51), sin embargo, en ambos casos, las TDxV eran inferiores en mujeres que en hombres (médicas 4,92 vs 5,48 y médicos 4,54 vs 4,93)

El mayor número de derivaciones se produjo a traumatología $(18,33 \%)$, oftalmología $(14,41 \%)$ y dermatología $(13,27 \%)$, el resto de especialidades no alcanzaban el $8 \%$ del total.

En el modelo ajustado del análisis multinivel (tabla 4) se observó que por cada año que aumentaba la edad media de las personas del cupo crecía 1\% la TDxV, los DDSS 2, 3 y 4 derivaron el $23 \%, 66 \%$ y $69 \%$, respectivamente, más que el DDSS 1. Ser paciente mujer disminuyó la TDxV en $88 \%$ y en $87 \%$ al ser atendidas por médico y médica respectivamente, es decir, las mujeres eran derivadas $12 \%$ y $13 \%$ menos que los hombres.

Como factor de efectos aleatorios, la razón de tasas del MF fue de 1,43\% indicando que la diferencia de tasas entre los MF que más y que menos derivaban era del $43 \%$.

Las tasas de derivación para cada díada en el estudio se presentan en la tabla 5, en la que se observa la ausencia de interacción sexo profesional-sexo paciente.

\section{Tabla 5}

Tasas de derivaciones por 100 visitas, ajustadas por las variables presentes en el modelo, según el sexo de profesional $y$ el sexo de paciente

\begin{tabular}{|l|c|c|c|}
\hline & $\begin{array}{c}\text { Paciente } \\
\text { hombre }\end{array}$ & $\begin{array}{c}\text { Paciente } \\
\text { mujer }\end{array}$ & $\mathrm{p}$ \\
\hline Médico & 4,61 & 4,21 & 0,000 \\
\hline Médica & 4,78 & 4,31 & 0,000 \\
\hline $\mathrm{p}$ & 0,871 & 0,872 & \\
\hline
\end{tabular}

\section{DISCUSIÓN}

Médicas y médicos presentaron mayores TDxC en mujeres y mayores TDxV en hombres. No se observaron diferencias de género en la práctica de MF pero los hallazgos sugieren que podrían existir desigualdades de género, sin que exista interacción sexo profesional-sexo paciente.

La gran variabilidad de tasas de derivación en los estudios en parte se debe a la diferente definición utilizada ${ }^{7}$, lo que limita las comparaciones.

En nuestro medio la TDxV fue 4,9, es decir, similar ${ }^{10,19,20} \mathrm{o}$ inferior a otros hallaz$\operatorname{gos}^{4,6,7,12}$. Se ha señalado que $\operatorname{los} /$ las $\mathrm{MF}$ resuelven en torno al $95 \%$ de los problemas de su consulta ${ }^{20,21}$ y se observa que los sistemas de salud basados en MF como gatekeeper presentan tasas de derivación simila$\mathrm{res}^{22,23}$. Parece que la intervención de especialistas se relaciona negativamente con la salud de la población ${ }^{24} \mathrm{y}$, aunque no es posible establecer estándares sobre la tasa de derivación idónea, cuando es inferior al 5\% muestra que las/los MF hacen su función de filtro de forma adecuada ${ }^{3}$.

En el análisis bivariante observamos que tanto médicas y médicos derivan más a mujeres cuando se ajusta por cupo, pero al ajustar por visitas ambos derivan más a los hombres.

El modelo de regresión identificó las variables asociadas a la TDxV. Como en otros estudios, las derivaciones no se asociaron a la formación del $\mathrm{MF}^{2}$ aunque la formación postgrado incrementa las derivaciones según una revisión ${ }^{5}$ y según otros estudios las disminuye ${ }^{7,22}$. Se ha argumentado que los esfuerzos en la formación médica no han disminuido el número de derivaciones ${ }^{6}$ pero hay que considerar que los patrones de práctica son también sensibles a la influencia de los iguales y de los aspectos organizativos, pudiendo todo ello contribuir a la 
ausencia de diferencias en las derivaciones. A diferencia de otros estudios ${ }^{2,7}$ la edad de MF no guarda relación con las derivaciones.

En los DDSS 2, 3 y 4 se produjeron mayores TDxV que en el DS 1, lo cual puede atribuirse, al menos en parte, a que las/os MF en este DDSS tienen mayor accesibilidad a pruebas diagnósticas, tanto en el propio CS como en la posibilidad de solicitarlas directamente. Este mayor acceso podría disminuir los riesgos para la salud de las personas que acuden a sus consultas $^{3}$. Aunque según otros estudios la distancia al nivel especializado influye en la tasa de las derivaciones ${ }^{2,4,13,25,26}$, no ocurre así en nuestro caso, en el que tampoco otras características del CS se asociaban a la tasas de derivaciones.

Nuestros objetivos estaban centrados en conocer la influencia del sexo de profesional y paciente en las derivaciones así como la existencia de interacción. El análisis multinivel mostró que el sexo de MF no influye. Otros estudios han encontrado tasas superiores de derivaciones en las médicas, tanto en España ${ }^{4}$ como fuera ${ }^{2,7,22,26-28}$. Como posibles causas se han esgrimido la menor tolerancia a la incertidumbre de las médi$\operatorname{cas}^{4,22,26}$, su mayor meticulosidad o menor autoconfianza ${ }^{2}$ y su diferente estilo de práctica $^{22,26}$. Las diferencias de práctica entre MF según género ${ }^{8,9}$ se atribuyen, fundamentalmente, a las diferencias en la socialización debidas a la construcción de género, y han dado lugar a debates sobre el impacto que tendrán dada la feminización del ejercicio de la medicina ${ }^{29}$. Puede ser que en Andalucía la formación y la organización del trabajo en atención primaria estén contribuyendo a homogeneizar la práctica de las derivaciones entre MF de ambos sexos.

En nuestro entorno había características profesionales, diferentes a las controladas en el modelo, que afectaban la variabilidad de las derivaciones, como expresa que el efecto de MF, tomado como factor de efec- tos aleatorios, fuera del $43 \%$, siendo esa la diferencia de tasas entre MF.

El análisis de las derivaciones por sexo del paciente mostró que, ajustando por tamaño del cupo, las mujeres fueron más derivadas que los hombres, pero al ajustar por visitas la tasa pasó a ser ligeramente superior en los hombres, resultados son sugerentes de posibles desigualdades de género.

En el análisis multinivel para la $\mathrm{TDxV}$, además del incremento de las derivaciones asociado a la edad del paciente, coherente con hallazgos previos ${ }^{2,11,26,30}$, observamos que las mujeres tienen menor probabilidad de ser derivadas que los hombres, concretamente del $88 \%$ y del $87 \%$ para médicos y médicas respectivamente. Numerosos estudios concuerdan con este resultado ${ }^{10,14,19,22,30} \mathrm{y}$, como ocurre en nuestro estudio, solo en las investigaciones que analizan las derivaciones por paciente -TDxC-se observan mayores tasas en las mujeres $^{2,11,13,26}$. También en Finlandia se observó que aunque se derivaban más mujeres la TDxV fue superior en los hombres, apoyando la idea de que el juicio médico es que los hombres consultan menos pero por motivos más serios ${ }^{22}$.Un estudio con derivaciones a otorrinolaringología mostró que ajustando por diagnóstico y comorbilidad las mujeres fueron menos derivadas a pesar de que consultaron más, concluyendo los autores que los/las MF pueden sesgar su práctica por considerar más graves los problemas que presentan los hombres ${ }^{30}$. En el seguimiento de casi 130.000 pacientes para evaluar desigualdades sociales analizó las derivaciones por dolor de cadera, dispepsia y sangrado posmenopáusico ${ }^{14}$, los resultados mostraron desigualdades por edad, estatus económico y género, ya que las mujeres fueron menos derivadas por dolor de cadera, lo que podría deberse, por un lado, a su mayor reticencia a operarse (por vivir con más frecuencia solas y/o ser responsables del cuidado familiar) y, por otro, a la existencia de sesgos en el sis- 
tema sanitario que comporta menores cuidados secundarios para las mujeres ${ }^{14}$. También en población española mayor de 60 años se ha notificado desigualdad en el uso de servicios sanitarios tras ajustar por número de enfermedades crónicas y calidad de vida, resultando que las mujeres consultan a su MF igual que los varones pero son menos hospitalizadas $^{31}$.

A pesar de que no conocemos cual sería la tasa de derivaciones adecuada, sí podemos decir que, globalmente, no hay razones para pensar que deba ser diferente según el sexo del paciente, pudiendo suponer el resultado encontrado un menor esfuerzo diagnóstico y/o terapéutico en las mujeres $y$, por tanto, la existencia de inequidad hacia ellas.

Considerando que en el estudio fueron excluidas las derivaciones a gineco-obstetricia, las especialidades a las que más se deriva coinciden o son muy similares a los hallazgos de estudios realizados en Espa$\tilde{\text { na }}{ }^{6,12,32}$ y en otros países ${ }^{13,20}$, mostrando cierto grado de homogeneidad y coherencia en la práctica de la medicina de familia.

Algunos estudios han encontrado asociación entre sexo de profesional y sexo de paciente en diferentes aspectos de la prácti$\mathrm{ca}^{28,33}$, sin embargo en nuestro entorno el análisis de las derivaciones por díadas no arrojó esta conclusión, las mujeres fueron menos derivadas en igual medida por médicos y médicas.

La mayor limitación del estudio fue que, desafortunadamente, la base de datos sobre derivaciones que se utiliza en el Sistema Sanitario Público en Andalucía no ofrece información clínica sobre diagnóstico y comorbilidad, los cuales son los principales determinantes de la tasa de derivacio$n^{1,11,24}$. La ausencia sobre información clínica y sobre la preferencia del paciente impide afirmar que exista inequidad, aunque la menor tasa de derivaciones observada en las mujeres, utilizando una base poblacional importante, sugiere la posible existencia de infrautilización de cuidados secundarios por parte de las mujeres, hecho conocido, si bien es un fenómeno que se deberá seguir investigando.

La principal ventaja del estudio fue que analiza las derivaciones durante un año registradas en una base de datos poblacional del sistema sanitario público en Andalucía, siendo el primero de esta magnitud en nuestro medio, lo que supone que los resultados tienen una elevada validez externa.

Seria pertinente desarrollar estudios que incorporen la información clínica. Así mismo, es necesario estudiar variables psicológicas de MF y pacientes ${ }^{5}$ ya que sabemos que existe una influencia recíproca entre MF y paciente, así la preferencia de los pacientes se relaciona con la conducta del $\mathrm{MF}^{34}$ y la percepción del paciente está influida por la interacción con su $\mathrm{MF}^{14}$.

Podemos concluir que no existen diferencias de práctica entre médicos y médicas de familia en las derivaciones realizadas desde la atención primaria de Andalucía, aunque existen indicios de la existencia de desigualdades de género en esta prestación de cuidados por parte del sistema sanitario, siendo las mujeres menos derivadas.

\section{BIBLIOGRAFÍA}

1. Mehrotra A, Forrest C, Lin C. Dropping the baton: specialty referrals in the United States. Milbank Q. 2011; 89:39-68.

2. Chan B, Austin P. Patient, physician, and community factors affecting referrals to specialists in Ontario, Canada. Med Care. 2003; 41: 500-11.

3. Gérvas J, García Olmos LM, Simó J, Peiró S, Seminario de Investigación 2007. Paradojas en la derivación de primaria a especializada. Aten Primaria. 2008; 40: 253-5.

4. García Olmos L, Abraira V, Gervas J, Otero A, Pérez Fernández M. Variability in GPs'referral rates in Spain. Fam Pract. 1995; 12: 159-62. 
5. O'Donnell CA. Variation in GP referral rates: what can we learn from the literature? Fam Pract. 2000; $17: 462-71$.

6. Prado Prieto L, García Olmos L, Rodríguez Salvanes F, Otero Puime A. Evaluación de la demanda derivada en atención primaria. Aten Primaria. 2005; 35: 146-51.

7. Ringberg U, Fleten N, Deraas T, Hasvold T, Forde $\mathrm{OH}$. High referral rates to secondary care by general practitioners in Norway are associated with GPs'gender and specialist qualifications in family medicine, a study of 4350 consultations. BMC Health Serv Res. 2013; $13: 147$.

8. Roter D, Hall J. Physician gender and patient-centered communication: a critical review of empirical research. Annu Rev Public Health. 2004; 25: 497-519.

9. Delgado Sánchez A. El papel del género en la relación médico-paciente. FMC. 1999; 6: 509-516.

10. Forrest C, Nutting P, Von Schrader S, Rohde C, Starfield B. Primary care physician specialty referral decision making: patient, physician, and health care determinants. Med Decis Making. 2006; 26: 76-85.

11. Sullivan C, Omar R, Ambler G, Majeed A. Casemix and variation in specialists referrals in general practice. Br J Gen Pract. 2005; 55: 529-33.

12. Sicras-Mainar A, Serrat-Tarrés J, Navarro-Artieda R, Llausí-Sellés R, Ruano-Ruano I, González-Ares JA. Adjusted clinical groups use as a measure of the referrals efficiency from primary care to specialized in Spain. Eur J Public Health. 2007; 17: 657-63.

13. Shadd J, Ryan B, Maddocks H, Thind A. Patterns of referral in a Canadian primary care Electronic health record database: retrospective cross-sectional analysis. Inform Prim Care. 2011; 19: 217-23.

14. McBride D, Hadroon S, Walters K, Gilmour S, Raine R. Explaining variation in referral from primary to secondary care: cohort study. BMJ. 2010; 341: c5267

15. Ruiz-Cantero MT. Sesgos de género en la atención sanitaria. 1ra ed. Granada: Escuela Andaluza de Salud Pública; 2009. 78 p.

16. Ruiz-Cantero MT, Ronda E, Alvarez-Dardet C. The importance of study design strategies in gender bias research: the case of respiratory disease management in primary care. J Epidemiol Community Health. 2007; 61: ii11-16.
17. Aguado-Romeo MJ. Márquez-Calderón S. BuzónBarrera M. Diferencias entre mujeres y varones en el acceso a procedimientos cardiovasculares intervencionistas en los hospitales públicos de Andalucía. Rev Esp Cardiol 2006. 59; 785:93.

18. Kempner J. Gendering the migraine market: do representations of illness matter? Soc Sci Med. 2006; 63: 1986-97.

19.Forrest C, Reid R. Prevalence of health problems and primary care physicians'speciality referral decisions. J Fam Pract. 2001; 50: 427-32.

20. Forrest C, Nutting P, Starfied B, Von Schader S. Family physicians'referral decisions. Results from the ASPN Referral Study. J Fam Pract. 2002; 51: 215-22.

21. García Olmos L. La investigación sobre demanda derivada. Semergen. 2005; 31: 345-6.

22. Vehviläinen A, Kumpusalo E, Voutilainen S, Takala J. Does the doctors' professional experience reduce referral rates? Evidence from the Finnish referral study. Scand J Prim Health Care. 1996; 14: 13-20.

23. Forrest C. Primary care gatekeeping and referrals: effective filter or failed experience? BMJ. 2003; 326: 692-695.

24. Starfield B. La investigación en Atención Primaria. Semergen. 2003; 29: 7-16.

25. Iverson GD, Coleridge ST, Fulda KG, Licciardone JC. What factors influence a family physician's decision to refer a patient to a specialist? Rural Remote Health. 2005; 5:413.

26. Liddy C, Singh J, Kelly R, Dahrouge S, Taljaard M, Younger $\mathrm{J}$. What is the impact of primary care model type on specialist referral rates? A cross-sectional study. BMC Fam Pract. 2014; 15:22.

27. Franks P, Williams G, Zwanziger J, Mooney C, Sorbero M. Why do physicians vary so widely in their referral rates? J Gen Intern Med. 2000; 15: 163-68.

28. Franks P, Bertakis K. Physician gender, patient gender, and primary care. J Womens Health. 2003; 12: 73-80.

29. Levinson W, Lurie N. When most doctors are women: what lies ahead? Ann Inter Med. 2004; 141: 471-4.

30. Cohen S, Kim J, Roy N, Courey M. Factors influencing referral of patients with voice disorders from primary to otolaryngology. Laryngoscope. 2014; 124: $214-20$ 
31. Redondo-Sendino A, Guallar-Castillón P, Ramón Banegas JR, Rodríguez-Artalejo F. Gender differences in the utilization of health-care services among the older adult population of Spain. BMC Public Health. 2006; 6: 155 .

32. Rodríguez-Alcalá FJ, Chacón-Fuentes J, Esteban Tudela M, Valles Fernández N, López de Castro F, Sánchez Ramiro A. Motivos de interconsulta entre atención primaria y el segundo nivel. Aten Primaria. 2005; 36:137-43.

33. Boulis A, Long J. Gender differences in the practice of adult primary care physicians. J Womens Health. 2004; 13: 703-12.

34. Delgado A, López-Fernández LA, Luna JD, SalettiCuesta L, Gil N, Jiménez M. The role of expectations in preferences of patients for a female or male general practitioner. Patient Educ Couns. 2011;82:49-57. 